\title{
Stringent Response and AggR-Dependent Virulence Regulation in the Enteroaggregative Escherichia coli Strain 042
}

\author{
Mário Hüttener ${ }^{1}$, Alejandro Prieto ${ }^{1}$, Joan Espelt ${ }^{1}$, Manuel Bernabeu ${ }^{1}$ and \\ Antonio Juárez ${ }^{1,2 *}$ \\ 'Department of Genetics, Microbiology and Statistics, Faculty of Biology, University of Barcelona, Barcelona, Spain, \\ ${ }^{2}$ Institute for Bioengineering of Catalonia, The Barcelona Institute of Science and Technology, Barcelona, Spain
}

OPEN ACCESS

Edited by:

Dongsheng Zhou,

Beijing Institute of Microbiology and Epidemiology, China

Reviewed by:

Shivangi Agarwal, Northwestern University, United States

Grzegorz Wegrzyn

University of Gdańsk, Poland Nicola Holden,

James Hutton Institute, United Kingdom Vijay Sharma,

National Animal Disease Center (USDA - ARS), United States

${ }^{*}$ Correspondence: Antonio Juárez ajuarez@ub.edu

Specialty section: This article was submitted to

Infectious Diseases,

a section of the journal

Frontiers in Microbiology

Received: 19 December 2017 Accepted: 27 March 2018

Published: 10 April 2018

Citation: Hüttener M, Prieto A, Espelt J, Bernabeu M and Juárez A (2018)

Stringent Response and AggR-Dependent Virulence Regulation in the Enteroaggregative

Escherichia coli Strain 042.

Front. Microbiol. 9:717.

doi: 10.3389/fmicb.2018.00717
Virulence expression in the enteroaggregative Escherichia coli strain 042 requires the transcriptional activator AggR. We show in this report that, as reported for other virulence factors, the nucleotide second messenger (p)ppGpp is needed for a high expression level of AggR. As expected from these findings, expression of AggR-activated genes such as the AafA pilin subunit is downregulated in the absence of (p)ppGpp. Considering the fact that biofilm formation in strain 042 requires the AafA protein, biofilm development in strain 042 is impaired in derivatives that lack either the AggR protein, the virulence plasmid that encodes AggR (pAA2) or the ability to synthesize (p)ppGpp. These results show a direct correlation between (p)ppGpp, expression of AggR and biofilm development in strain 042.

Keywords: (p)ppGpp, AggR, EAEC, biofilm, AAF/II

\section{INTRODUCTION}

Enteroaggregative Escherichia coli (EAEC) strains are diarrheal pathogens (Kaper et al., 2004). The EAEC strains can be distinguished from enteropathogenic E. coli (EPEC) because of their different patterns of adherence to HEp-2 cells. Whereas EPEC display a "microcolony" pattern of adherence, EAEC display a characteristic aggregative or "stacked-brick" pattern (Nataro et al., 1987). EAEC adherence to intestinal cells is mediated by fimbrial adhesins, designated aggregative adherence fimbriae (AAFs).

Epidemiological studies have shown that EAEC strains are genetically heterogeneous. Hence, a large number of virulence factors have been identified in EAEC clinical isolates (Okeke et al., 2010). The strain O104:H4 is an example of EAEC genetic heterogeneity. It caused a few years ago in Germany a large outbreak of bloody diarrhea (Frank et al., 2011). Isolates from the O104:H4 outbreak harbor a plasmid (pAA) that encodes, among other virulence factors, the fimbriae that mediate the EAEC type of adherence (Bielaszewska et al., 2011). Nevertheless, unlike typical EAEC strains, strain O104:H4 contains a prophage encoding the Shiga toxin (Mayer et al., 2012), which is a well-characterized virulence determinant usually expressed by a different $E$. coli pathotype, enterohemorrhagic E. coli (Nataro and Kaper, 1998). The prototypical strain for the study of EAEC virulence factors and pathogenicity is strain 042, which caused diarrhea in a volunteer trial (Nataro et al., 1995). Its genome sequence is available (Chaudhuri et al., 2010) and the virulence factors are characterized. Strain 042 harbors the IncFIC virulence plasmid pAA2 (Nataro et al., 1985; 
Chaudhuri et al., 2010), which codes, among other genes, for the fimbrial adhesion determinants (the AAF/II variant of $\mathrm{AAF}$ ), for the virulence master regulator AggR and for other virulence determinants (Nataro et al., 1994; Czeczulin et al., 1997; Chaudhuri et al., 2010; Morin et al., 2010). The AggR protein belongs to the family of the AraC-like transcriptional activators and is encoded by many EAEC strains. AggR activates it is own expression (Morin et al., 2010) and also the expression of several EAEC virulence factors encoded in the pAA2 plasmid (Morin et al., 2013), including the surface protein dispersin Aap (Sheikh et al., 2002) and the genes responsible for the synthesis of the AAF/II fimbriae (Elias et al., 1999). AggR also regulates the expression of some chromosomally encoded virulence determinants, such as a type VI secretion system identified in strain 042 (Morin et al., 2013). Details of $\operatorname{aggR}$ regulation are available. Regulation occurs both at the transcriptional and post-transcriptional levels. $a g g R$ transcription is repressed by the nucleoid associated protein H-NS (Morin et al., 2010). The FIS protein is also required for $a g g R$ expression (Sheikh et al., 2001). Post-transcriptional repression of AggR expression by the Aar protein (an AraC-member of negative regulators) has been recently described (Santiago et al., 2014).

The nucleotide second messenger (p)ppGpp drives the stringent response in bacteria (as reviewed in Dalebroux et al., 2010). This alarmone also plays relevant roles in bacterial persistence and virulence (Dalebroux et al., 2010; Maisonneuve et al., 2013). Classical examples of (p)ppGpp modulating bacterial virulence include, among others, adherence of enterohemorrhagic and uropathogenic E. coli strains (Aberg et al., 2006, 2008, 2009; Nakanishi et al., 2006) or invasion in S. enterica serovar Typhimurium (Pizarro-Cerdá and Tedin, 2004; Song et al., 2004). In this work we present new data regarding AggR regulation. We show that (p)ppGpp is required for proper expression of this transcriptional activator. According to this observation, the expression of AggR-activated virulence determinants and the formation of biofilm are significantly impaired in a (p)ppGpp ${ }^{0}$ mutant derivative of strain 042 .

\section{MATERIALS AND METHODS}

\section{Bacterial Strains, Plasmids, and Culture Media}

All bacterial strains and plasmids used in this work are listed in Table 1. Cultures were routinely grown in Luria Broth (LB) medium (10 g NaCl, $10 \mathrm{~g}$ tryptone, and $5 \mathrm{~g}$ yeast extract per liter) with vigorous shaking at $200 \mathrm{rpm}$ (Innova 3100, New Brunswick Scientific). Antibiotics were used at the following concentrations: kanamycin $(\mathrm{Km})\left(50 \mu \mathrm{g} \mathrm{ml}^{-1}\right)$, chloramphenicol (Cm) $\left(25 \mu \mathrm{g} \mathrm{ml}^{-1}\right)$, carbenicillin (Cb) $\left(100 \mu \mathrm{g} \mathrm{ml}^{-1}\right)$.

To construct plasmids pBAD-AggR and pBR322-SpoT, the aggR and spoT genes from strain E. coli 042 were amplified using oligonucleotides AggREcoRI18.5-AggRXbaI18.3 and spoT042pbr322ECORIfw5-spoT042pbr322BAMHIrev3 (see Supplementary Table S1, for the sequence) together with Phusion Hot Start II High-Fidelity DNA Polymerase (Thermo
Scientific) following the manufacturer's recommendations. aggR and spoT amplification with the above referred oligonucleotides generates EcoRI/XbaI and EcoRI/BamHI sites flanking the $a g g R$ and spoT genes, respectively. The corresponding EcoRI/XbaI and EcoRI/BamHI fragments were cloned into the vector pBAD18 and pBR322 digested with the same enzymes. The resulting plasmid was termed pBAD-AggR and pBR322SpoT.

\section{Genetic Manipulations}

All enzymes used to perform standard molecular and genetic procedures were used according to the manufacturer's recommendations. To introduce plasmids in E. coli, bacterial cells were grown until an $\mathrm{OD}_{600 \mathrm{~nm}}$ of 0.6. Cells were then washed several times with $10 \%$ glycerol, and the respective plasmids were electroporated by using an Eppendorf gene pulser (Electroporator 2510).

TABLE 1 | Bacterial strains and plasmids used in this study.

\begin{tabular}{|c|c|c|}
\hline Bacterial strains & Description & $\begin{array}{l}\text { Source or } \\
\text { reference }\end{array}$ \\
\hline 042 & E. coli EAEC, $\mathrm{Cm}^{r} \mathrm{Sm}^{r} \mathrm{Tc}^{r}$ & Prof. I. Henderson \\
\hline 042LC & $042 \Delta / a c Z \Delta c a t$ & This work \\
\hline 042LCaggRlacZ & 042LC aggR::lacZ & This work \\
\hline 042LCaggRlacZrelA & 042LC aggR::lacZ $\Delta$ relA & This work \\
\hline 042LCaggRlacZrelAspoT & 042LC aggR::lacZ $\Delta$ relA $\Delta s p o T$ & This work \\
\hline 042aggR & 042 aggR::km & This work \\
\hline 042relAspoT & $042 \Delta r e l A \Delta s p o T$ & This work \\
\hline 042AggRFlag & 042 AggR-Flag & This work \\
\hline 042AafAFlag & 042 AafA-Flag & This work \\
\hline 042AggRFlagrelA & 042 AggR-Flag $\Delta$ relA & This work \\
\hline 042AafAFlagrelA & 042 AafA-Flag $\Delta$ relA & This work \\
\hline 042AggRFlagrelAspoT & 042 AggR-Flag $\Delta$ relA $\Delta s p o T$ & This work \\
\hline 042AafAFlagrelAspoT & 042 AafA-Flag $\Delta$ relA $\Delta s p o T$ & This work \\
\hline 042pAA ${ }^{-}$ & 042 Cured from pAA2 plasmid & This work \\
\hline $\mathrm{DH} 5 \alpha$ & $\begin{array}{l}\text { E. coli, fhuA2 lac(del)U169 phoA } \\
\text { glnV44 } \Phi 80^{\prime} \text { lacZ(del)M15 } \\
\text { gyrA96 recA1 relA1 endA1 thi-1 } \\
\text { hsdR17 }\end{array}$ & Taylor et al., 1993 \\
\hline
\end{tabular}

\begin{tabular}{|c|c|c|}
\hline Plasmids & Description & $\begin{array}{l}\text { Source or } \\
\text { reference }\end{array}$ \\
\hline pBAD18 & rep $_{\mathrm{pMB} 1} \mathrm{p}_{\mathrm{araBAD}} \mathrm{Cb}^{r}$ & $\begin{array}{l}\text { Guzman et al., } \\
1995\end{array}$ \\
\hline pBAD-AggR & pBAD18 + aggR from EAEC 042 & This work \\
\hline pBR322 & ori $i_{p} \mathrm{MB} 1, \mathrm{Tc}^{r}, \mathrm{Ap}^{\mathrm{r}}$ & Bolivar et al., 1977 \\
\hline pBR322-SpoT & pBR322 + spoT from EAEC 042 & This work \\
\hline pKD3 & oriR $\gamma, \mathrm{Cm}^{r}, \mathrm{Ap}^{r}$ & $\begin{array}{l}\text { Datsenko and } \\
\text { Wanner, } 2000\end{array}$ \\
\hline pSUB11 & $\begin{array}{l}\text { FLAG- and } \mathrm{Km}^{\mathrm{r}} \text {-coding template } \\
\text { vector }\end{array}$ & Uzzau et al., 2001 \\
\hline pKD4 & oriR $\gamma, \mathrm{Km}^{r}, \mathrm{Ap}^{r}$ & $\begin{array}{l}\text { Datsenko and } \\
\text { Wanner, } 2000\end{array}$ \\
\hline pKD46 & $\begin{array}{l}\text { oriR101, repA101 (ts), } \\
\text { AraBp-gam-bet-exo }\end{array}$ & $\begin{array}{l}\text { Datsenko and } \\
\text { Wanner, } 2000\end{array}$ \\
\hline pCP20 & $\lambda$ cl857 (ts), ts-rep (FLP ts) & $\begin{array}{l}\text { Cherepanov and } \\
\text { Wackernagel, } 1995\end{array}$ \\
\hline
\end{tabular}


The WT 042 strain is $\mathrm{Cm}$ resistant $\left(\mathrm{Cm}^{\mathrm{r}}\right)$ and encodes the lacZ gene. To construct an aggR::lacZ transcriptional fusion in this strain we needed first to knock out the lac $Z$ gene. Taking into account that $\mathrm{Km}$ resistance $\left(\mathrm{Km}^{\mathrm{r}}\right)$ is conferred by the genetic approach used to generate the lac $Z$ transcriptional fusion, we needed a second marker for selection of additional mutations. Hence, we decided to inactivate the cat gene as well. Upon obtaining the lac $Z$ cat derivative of strain 042 (termed 042LC) we used it to generate a transcriptional lacZ fusion on the aggR gene, generating strain 042LCaggRLacZ (aggR::lacZ).

Since inactivation of relA and spoT genes in strain 042 is a previous step to evaluate the role of (p)ppGpp on expression of the $\operatorname{aggR}$ gene, we knocked out both genes in strain 042 and in different mutant derivatives. To that end, we fist knocked out the relA gene in strains WT 042, 042LCaggRLacZ, 042AggRFlag, and 042AafAFlag. First, a $\mathrm{Cm}^{\mathrm{r}}$ derivative was obtained by the $\lambda$ Red recombinant method in the relA locus and then, we took advantage of FLP recombinase encoded by $\mathrm{pCP} 20$ plasmid to eliminate the $\mathrm{Cm}^{\mathrm{r}}$ cassette, generating the corresponding $\triangle$ relA isogenic mutants. Thereafter, the spoT gene was deleted from the $\Delta$ relA derivatives of the different strains by the $\lambda$ Red recombinant method inserting a $\mathrm{Cm}^{\mathrm{r}}$ cassette in the spoT locus. Again, pCP20 plasmid was used to eliminate the $\mathrm{Cm}^{\mathrm{r}}$ determinant, generating the $\Delta$ relA $\Delta$ spoT mutant derivative in the strains; WT 042 (042relAspoT), 042LCaggRLacZ (042LCaggRlacZrelAspoT), 042AggRFlag (042AggRFlagrelAspoT), and 042AafAFlag (042AafAFlagrelAspoT), respectively.

To obtain the above referred mutant derivatives lacking lac $Z$, cat, $\operatorname{aggR}$, relA, and spoT alleles in the EAEC strain 042, the $\lambda$ Red recombinant method described by Datsenko and Wanner (2000) was used. Briefly, the $\mathrm{Km}^{\mathrm{r}}$ cassette of plasmid pKD4 was amplified using oligonucleotides LacZ042P1/LacZ042P2, Cat042P1/Cat042P2, and AggR042P1/AggR042P2 for lacZ, cat, and $\operatorname{aggR}$ deletions, respectively (see Supplementary Table S1, for the corresponding sequences). For mutation in the alleles relA and spoT, the $\mathrm{Cm}^{\mathrm{r}}$ cassette from plasmid pKD3 was amplified using respectively oligonucleotides RelA042P1/RelA042P2 and SpoT042P1/SpoT042P2 (see Supplementary Table S1, for the corresponding sequences). DNA templates were treated with DpnI (Thermo Scientific) following manufacturer recommendations and then, purified and electroporated to the competent cells. Mutants were selected on LB plates containing the appropriate selection marker ( $\mathrm{Km}$ or $\mathrm{Cm}$ ) and the successful deletion of the corresponding gene was confirmed by PCR using the primers $\mathrm{KT}$ or Cat- $\mathrm{C} 1\left(\mathrm{Km}^{\mathrm{r}}\right.$ and $\mathrm{Cm}^{\mathrm{r}}$, respectively) in combination with specific primers located in the remaining gene sequence in the bacterial chromosome (see Supplementary Table S1, for the corresponding sequences).

When necessary, the antibiotic resistance cassette was eliminated by transforming the mutant strain with plasmid pCP20 and subsequent incubation at $42^{\circ} \mathrm{C}$ for two or more passages as reported (Datsenko and Wanner, 2000). The pCP20 plasmid encodes the Flp recombinase that catalyzes the recombination between the FRT sites flanking the antibiotic resistance cassettes (Cherepanov and Wackernagel, 1995). The
FRT-generated site in the gene $\operatorname{aggR}$ was used to integrate plasmid pKG136 (Ellermeier et al., 2002), thereby generating the transcriptional aggR::lacZY fusion.

Insertions of FLAG sequences to the $\operatorname{aggR}$ and aafA genes were obtained by a modification of the $\lambda$ Red recombinant method, as described by Uzzau et al. (2001). The antibiotic-resistance determinant of plasmid pSUB11 was amplified using oligonucleotides AggR3xP1/AggR3xP2 and AafA3xP1/AafA3xP2 for the aggR and aafA genes, respectively (see Supplementary Table S1, for the corresponding sequences). Mutants were selected on LB plates containing $\mathrm{Km}$, and successful FLAG insertion was confirmed by PCR using the oligonucleotides $\mathrm{KT}\left(\mathrm{Km}^{\mathrm{r}}\right)$ in combination with specific oligonucleotides located in the remaining gene sequence nearby (see Supplementary Table S1, 3xP1UP/3xP2DOWN series oligonucleotides). The chromosomal fusions AggR-Flag and AafA-Flag were constructed in the parental strain E. coli 042. The $\triangle$ relA and $\triangle$ relA $\triangle$ spoT mutations were introduced in the strains encoding Flag-tag constructions as described above.

We used strain 042AggRFlag, which contains a Flag-tag insertion at the $3^{\prime}$-end of the $\operatorname{aggR}$ gene and a kanamycin cassette, which confers resistance for selection, for a plasmid curing protocol. The protocol used was a modification of a previously reported (Hooper et al., 1984). Briefly, we started with an overnight culture of strain $042 \mathrm{AggRF}$ lag grown at $37^{\circ} \mathrm{C}$ in medium LB plus $50 \mu \mathrm{g} \mathrm{ml}^{-1} \mathrm{Km}$. Bacterial cells were reinoculated (1:1000) in fresh LB medium during 10 consecutive days. Serial dilutions were then prepared from the 10th overnight culture in LB medium and bacterial cells were spread in LB plates supplemented with novobiocin $\left(3,3 \mu \mathrm{g} \mathrm{ml}^{-1}\right)$. Resistant colonies were tested for loss of $\mathrm{Km}^{\mathrm{r}}$. Kanamycin sensitive colonies were subsequently tested for plasmid loss by PCR-detection of plasmid genes.

\section{Beta-Galactosidase Assay}

$\beta$-Galactosidase activity measurements were performed as described by Miller (1992). The Student's $t$-test was used to determine statistical significance, the values were obtained by using the GraphPad Prism 5 software. A P-value of less than 0.05 was considered significant.

\section{SDS-PAGE and Western Blotting}

Protein samples were analyzed by SDS-PAGE at $12.5 \%$ (Sambrook, 2001). Proteins were transferred from the gels to PVDF membranes using the Trans-Blot Turbo system (BioRad). Western blot analysis was performed with monoclonal antibody raised against the Flag-epitope (1:10.000 - Sigma) incubating $16 \mathrm{~h}$ at $4^{\circ} \mathrm{C}$. Membranes were washed three times of 20 min each with PBS $0.2 \%$ Triton solution. Thereafter they were incubated with horseradish peroxidase-conjugated goat antimouse IgG (1:2500 - Promega) during $1 \mathrm{~h}$ at room temperature. Again, membranes were washed three times of 20 min with PBS $0.2 \%$ Triton solution and detection was performed by enhanced chemiluminescence using Quantity One software (Bio-Rad). 


\section{Isolation of RNA}

Bacterial cells were grown until $\mathrm{OD}_{600} \mathrm{~nm}$ of $2.0 .5 \mathrm{ml}$ of cells were then mixed with 0.2 volume of stop solution buffer (95\% Ethanol, 5\% Phenol), shaken and centrifuged (10 min, $6,000 \times g$ ). Bacterial pellets were subsequently frozen at $-80^{\circ} \mathrm{C}$ until use. Total RNA was extracted from bacterial pellets using Tripure Isolation Reagent (Roche) according to the manufacturer's instructions. Potential traces of DNA were removed by digestion with DNase I (Turbo DNA-free, Ambion), according to the manufacturer's instructions. RNA concentration and RNA quality were measured using a Nano-Drop 1000 (Thermo Fisher Scientific).

\section{Quantitative Reverse Transcription-PCR (qRT-PCR)}

Expression levels of aggR, aafA, aafD, aat $P$, and aap genes were determined by using real-time quantitative PCR. Briefly, $1 \mu \mathrm{g}$ of previously isolated total RNA was reverse transcribed to generate cDNA using the High-capacity cDNA Reverse Transcription kit (Applied Biosystems) according to the manufacturer's instructions. All samples within an experiment were reverse transcribed at the same time; the resulting cDNA was diluted 1:100 in nuclease-free water and stored in aliquots at $-80^{\circ} \mathrm{C}$ until used. As a control, parallel samples in which reverse transcriptase was omitted from the reaction mixture, were run. Real-time PCR was carried out using Maxima SYBR green/ROX qPCR master mix (Thermo Scientific) and the ABI Prism 7700 sequence detection system (Applied Biosystems). Specific oligonucleotides complementary to the genes of interest were designed using primer3 software. The primers were named aggRRTFW/aggRRTRV, aafARTFW/aafARTRV, aafDRTFW/aafDRTRV, aatPRTFW/aatPRTRV, and aapRTFW/ aapRTRV for $\operatorname{aggR}$, aafA, aafD, aatP, and aap genes, respectively (see Supplementary Table S1, for the corresponding sequences). Relative quantification of gene expression of mutants versus wildtype strain was performed using the comparative threshold cycle (CT) method (Livak and Schmittgen, 2001). The relative amount of target cDNA was normalized using the gapA gene as an internal reference standard.

\section{Biofilm Quantification}

Biofilm assay and quantification were performed as described (Sheikh et al., 2001) with some modifications. Briefly, bacterial cells were grown overnight in $\mathrm{LB}$ medium at $37^{\circ} \mathrm{C}$ and then, re-inoculated (1:1000) in fresh $\mathrm{LB}$ medium and incubated at $37^{\circ} \mathrm{C}$ until an $\mathrm{OD}_{600}$ of 2.0 was reached. These cultures were used to inoculate $(1: 100)$ wells of Nuclon Delta Surface plates (24 and 96 wells - Thermo Scientific) containing LB medium supplemented with glucose at $0.45 \%$ of final concentration. Plates were incubated at $37^{\circ} \mathrm{C}$ for $16 \mathrm{~h}$. Biofilms were then washed twice with PBS and stained with 5\% crystal violet. Biofilms were solubilized in $95 \%$ ethanol and quantified spectrophotometrically at $570 \mathrm{~nm}$. The Student's $t$-test was used to determine statistical significance. The values were obtained by using the GraphPad Prism 5 software. A $P$-value of less than 0.05 was considered significant.

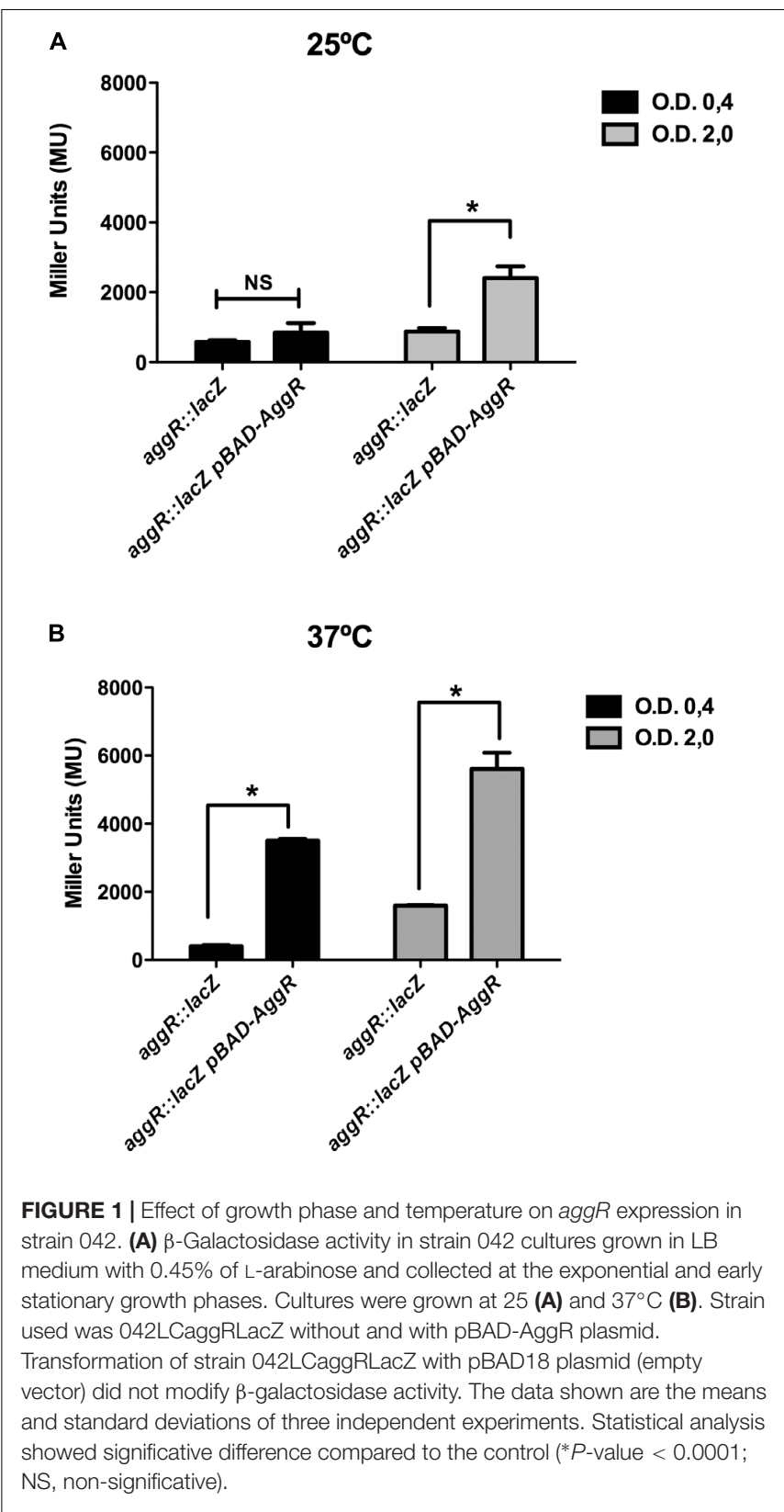

\section{RESULTS}

\section{Effect of Temperature and Growth Phase on agg $R$ Expression}

The aim of this study was to gain insight into the regulation of the $a g g R$ gene in strain 042. Upon generating an $\operatorname{aggR::lacZ}$ transcriptional fusion, we studied first the effect of temperature and growth phase on the expression of this transcriptional activator. Taking into account that AggR positively modulates its expression (Morin et al., 2010) and that the transcriptional fusion generated by us disrupts AggR, we provided the AggR protein in trans by transforming plasmid $\mathrm{pBAD}-\mathrm{AggR}$ in strain 042LCaggRLacZ. By doing this, we aimed to avoid that the 


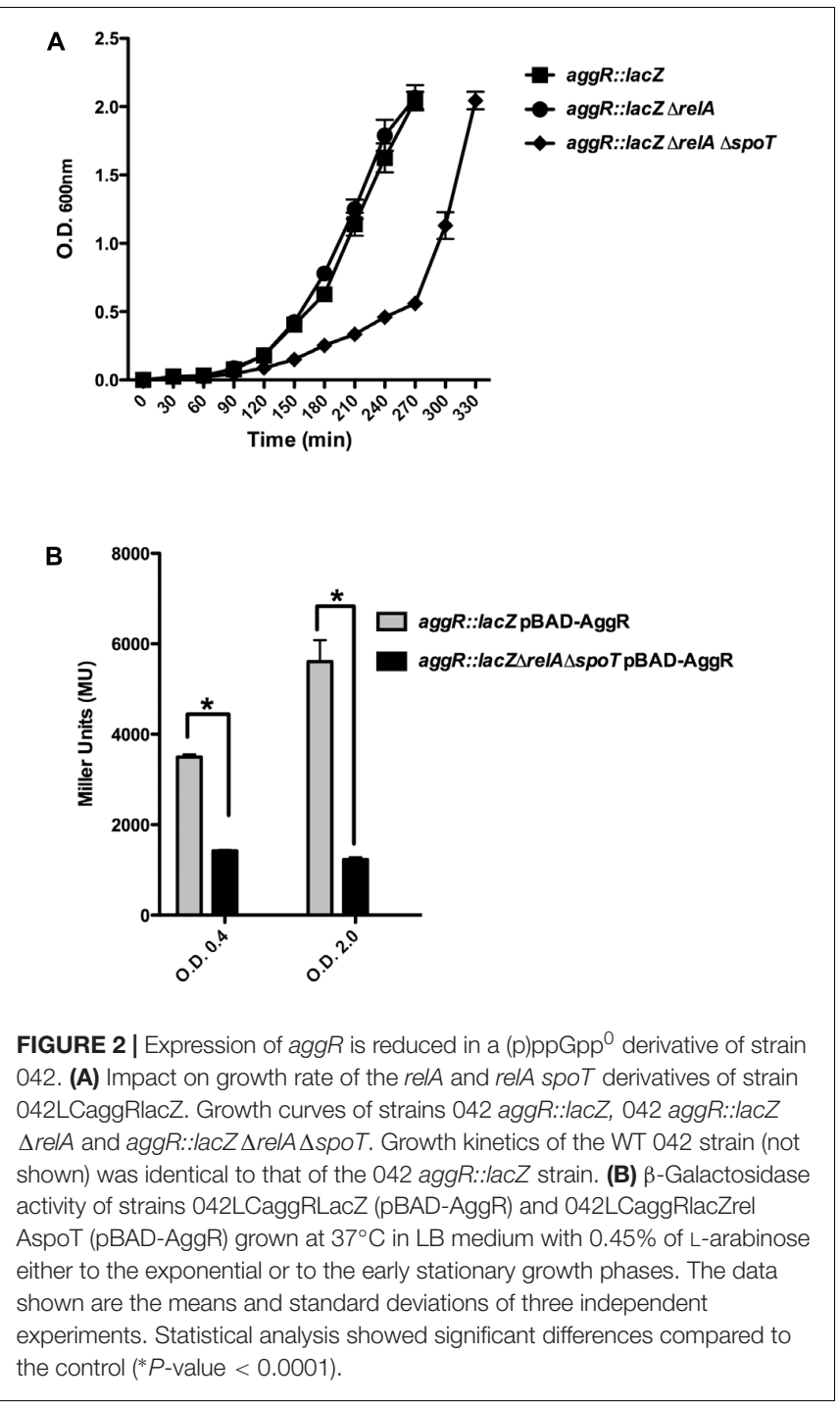

lack of AggR would mask or alter the effect of different growth conditions on lacZ transcription. Strains 042LCaggRlacZ and 042LCaggRLacZ (pBAD-AggR) were grown in LB medium either at 25 or at $37^{\circ} \mathrm{C}$, samples were taken both at the midlogarithmic growth phase $\left(\mathrm{OD}_{600}\right.$ of 0.4$)$ and at the onset of the stationary phase $\left(\mathrm{OD}_{600}\right.$ of 2.0$)$, and beta-galactosidase activity was determined. The results obtained showed that there exists growth phase- and temperature-dependent regulation of aggR (Figures 1A,B). The presence of plasmid pBAD-AggR showed that, as predicted. AggR activates it own transcription.

\section{agg $R$ Transcription Is Downregulated in a (p)ppGpp-Null Mutant Derivative of} Strain 042

Upon obtaining a double 042 relA spoT mutant, we assessed whether lack of (p)ppGpp influences growth and $\operatorname{aggR}$ expression in strain 042. Strains 042LCaggRlacZ and 042LCaggRlacZrelAspoT were used for these studies. Although $\operatorname{aggR}$ expression is higher when cells grow in DMEM medium

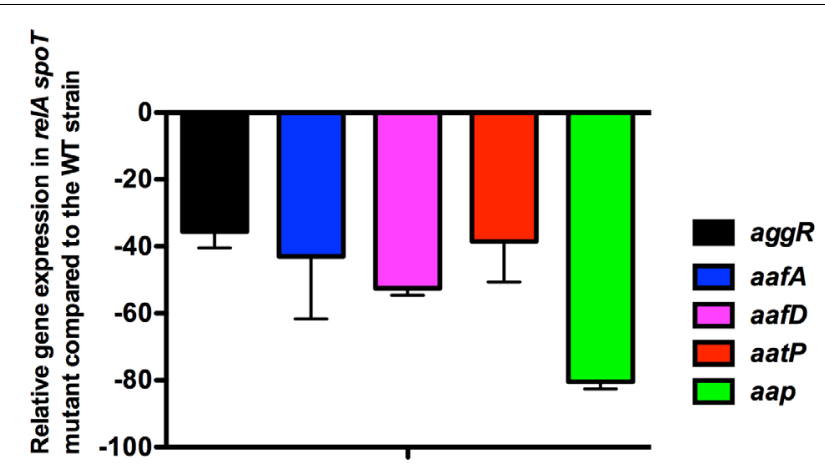

FIGURE 3 | Effect of the double relA spoT mutation on transcription of aggR, and AggR-activated genes in strain 042. Fold change values (qRT-PCR values) of aggR, and AggR-activated genes aafA, aafD, aatP, and aap in strain $042 \Delta$ relA $\Delta$ spoT. Expression of the corresponding genes in the 042 WT strain was considered as 1.0. The data shown are the means and standard deviations of three independent experiments are shown.

A

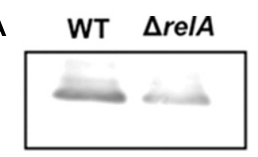

B

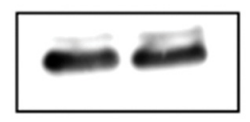

WT $\Delta$ relA $\Delta$ spoT
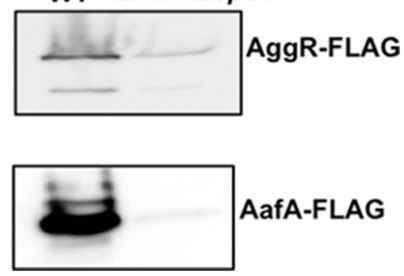

FIGURE 4 | Expression of AggR and AafA proteins in strain 042 is reduced in the absence of (p)ppGpp. Immunodetection of AggR-Flag (A) and AafA-Flag (B) proteins in total cell extracts from cultures from strain 042 and its isogenic mutants $\Delta r e / A$ and $\Delta r e / A \Delta s p o T$. Experiments were repeated three times. A representative experiment is shown. than in LB medium (Morin et al., 2010), we selected this latter in order to reduce the predicted impact of the double mutation relA spoT on the growth rate that cells harboring these mutations exhibit when growing in minimal media. Even growing in LB medium, the (p)ppGpp ${ }^{0}$ derivative of strain 042 (aggR::lacZ) showed a reduced growth rate (Figure 2A). To evaluate the role of (p)ppGpp on $a g g R$ expression, we measured first transcription of $a g g R$ as $\beta$-galactosidase activity. Again, both the $042 \mathrm{WT}$ and the relA spoT mutant strains were transformed with plasmid pBAD-AggR. When compared with strain 042LCaggRlacZ, strain 042LCaggRlacZrelAspoT showed significantly reduced $\beta$-galactosidase levels (Figure 2B). The results obtained by using the lac $Z$ transcriptional fusion were corroborated by specifically measuring $\operatorname{aggR}$ transcription by qRT-PCR (Figure 3). aggR expression is significantly downregulated in the (p)ppGpp ${ }^{0}$ mutant. We also used qRTPCR to measure (p)ppGpp-dependent transcription of some of the AggR-activated genes, namely aafA, aafD, aatP, and aap. aafA and aafD genes encode the proteins responsible for the biogenesis of the AAF/II fimbriae (being AafA the major subunit and AafD a chaperone of AAF/II, respectively). AatP (an inner-membrane permease) belongs to the cluster 

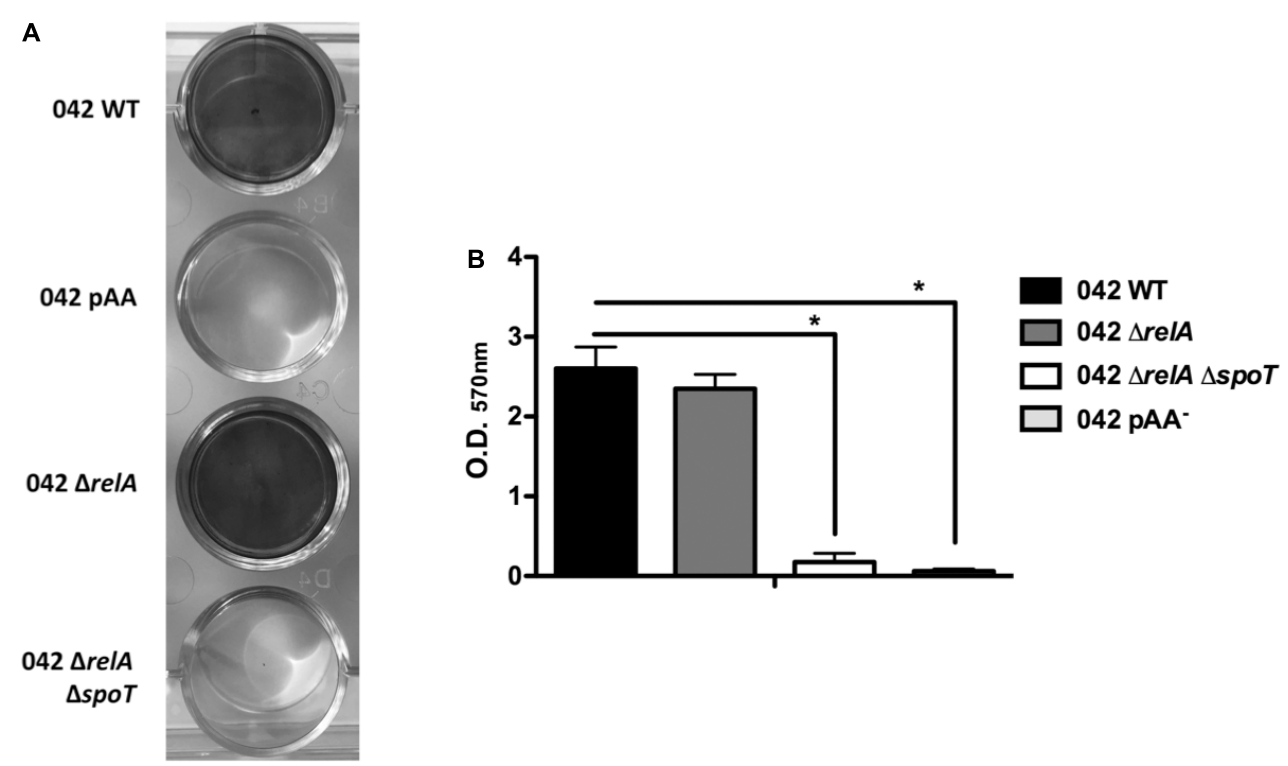

FIGURE 5 | Biofilm formation in the 042 strain is directly dependent on (p)ppGpp. (A) Crystal violet stain of biofilms formed by strains 042,042 pAA, $042 . \Delta$ relA, and $042 \Delta$ relA $\Delta$ spoT. (B) Biofilm quantification $\left(\mathrm{OD}_{570} \mathrm{~nm}\right)$. The data shown are the means and standard deviations of three independent experiments. Statistical analysis showed significant differences compared to the control ( $P$-value $<0.0001)$.

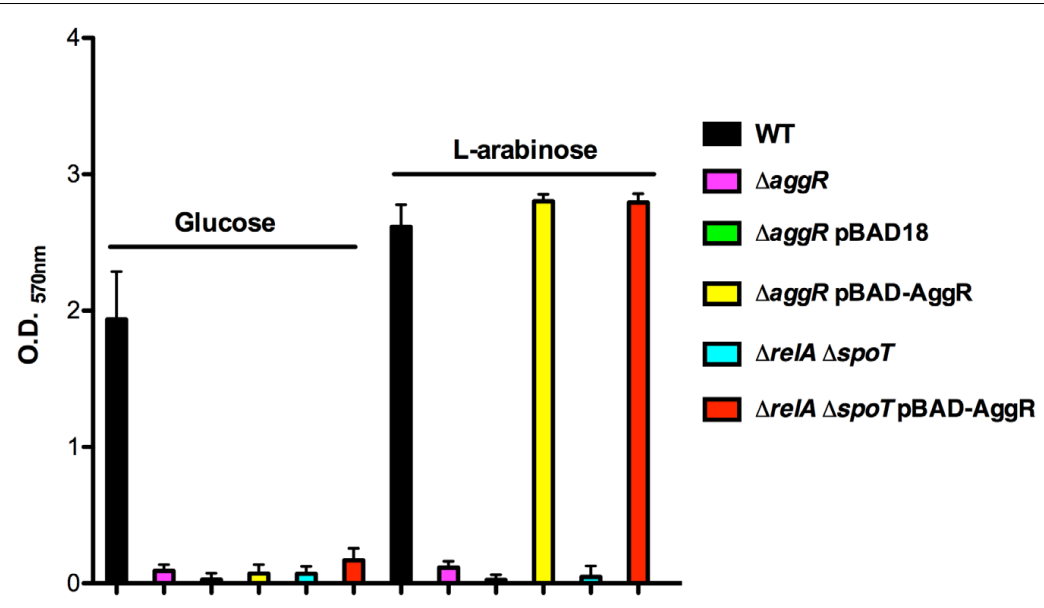

FIGURE 6 | Biofilm formation in strain 042 requires both the AggR protein and physiological levels of (p)ppGpp. Quantification of biofilm formed by strain 042 and its aggR and relAspoT derivatives, in the presence and in the absence of plasmid pBAD-AggR. Cells were cultured in LB medium supplemented either with glucose or $\mathrm{L}$-arabinose at $0.45 \%$. The data shown are the means and standard deviations of three independent experiments.

responsible for secretion of the dispersin protein (AaP), which promotes dispersal of EAEC across the intestinal mucosa. As expected, due to the reduced expression of AggR, those genes altogether are downregulated in absence of (p)ppGpp (Figure 3).

\section{AggR and AafA Proteins Show a (p)ppGpp-Dependent Expression}

To correlate transcriptional data with protein expression, $a g g R$ and aafA genes were Flag-tagged. Thereafter, Flag-tagged AggR and AafA proteins were immunodetected in total cell extracts, both in the WT 042 strain as well as in the corresponding relA and relA spoT derivatives. Culture conditions for AggR and AafA immunodetection were those used to measure aggR transcription [growth in $\mathrm{LB}$ medium at $37^{\circ} \mathrm{C}$ to the early stationary phase $\left(\mathrm{OD}_{600}\right.$ of 2.0)]. The results obtained are in accordance with the transcriptional data obtained. The levels of both AggR and AafA proteins are reduced in the relA spoT derivative (Figure 4, see Supplementary Figures S1-S3 to full Western blot membranes). The fact that they are not reduced in the relA mutant can be interpreted as spoT activity rendering (p)ppGpp levels high enough as to enable high-level expression of these proteins in strain 042. 


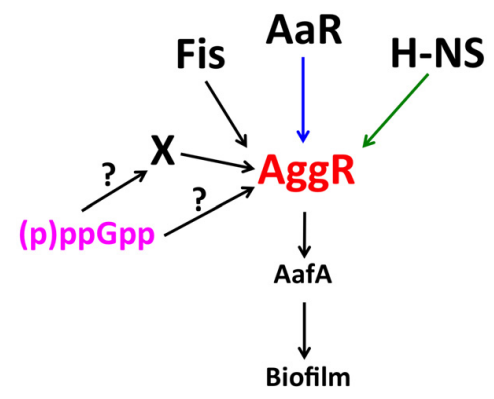

FIGURE 7 | Diagram showing the regulatory cascade influencing AggR expression. AggR is a key virulence regulator in EAEC. Downstream-regulated virulence factors include the AafA pilin subunit. FIS and $\mathrm{H}-\mathrm{NS}$ proteins respectively induce and repress aggR transcription (black and green arrows, respectively). AaR protein post-transcriptionally represses AggR protein expression (blue arrow). We show in this work that the (p)ppGpp alarmone is a key factor regulating either directly or indirectly expression of aggR and AggR-activated genes.

\section{(p)ppGpp Is Required for Biofilm Formation in the EAEC Strain 042}

It is well-established that $\operatorname{aggR}$ mutants are defective in biofilm formation in strain 042 (Sheikh et al., 2001). Downregulation of the major pilin subunit (AafA protein) is underlying that phenotype (Sheikh et al., 2001). Taking into account that, as shown above, AafA expression is dependent on proper (p)ppGpp levels, we hypothesized that biofilm formation should also be downregulated in a (p)ppGpp ${ }^{0}$ derivative of strain 042. To assess this, we measured biofilm formation in cells growing in LB-glucose $(0.45 \%$ final concentration). Strains analyzed were WT 042, 042relAspoT, and 042pAA ${ }^{-}$. As expected, biofilm production is drastically reduced both in the (p)ppGpp ${ }^{0}$ mutant as well as in the WT strain lacking pAA2 plasmid (Figures 5A,B). We also decided to test whether expression in trans of the AggR protein can complement that phenotype. To do this, plasmid pBAD-AggR was transformed in strains WT 042, 042aggR, and 042relAspoT. Cells were grown in LB medium supplemented either with glucose (negative control) or L-arabinose (AggR expression), and biofilm formation was assessed in the different strains (Figure 6). When cells were grown in conditions leading to specific AggR expression (LB plus L-arabinose), the presence of plasmid pBAD-AggR induced biofilm formation both in strains 042aggR and 042relAspoT, thus providing evidence for AggR being required for biofilm formation, and correlating (p)ppGpp and biofilm formation via AggR (Figure 6).

\section{DISCUSSION}

We bring in this paper new information about the physiological inputs that dictate AggR expression (and hence virulence expression) in the EAEC strain 042. As it happens with many other virulence factors in $E$. coli and other pathogens infecting warm-blooded hosts, virulence expression requires temperatures close to that of their hosts. Temperature-dependent expression of AggR is consistent with the reported effect of the nucleoid-associated protein $\mathrm{H}-\mathrm{NS}$ repressing its expression (Morin et al., 2010) (Figure 7). We also found that there is growth-phase dependent expression of $\operatorname{aggR}$ when cells grow in LB medium. A significant increase in $a g g R$ transcription occurs when cells enter the stationary phase $\left(\mathrm{OD}_{600}\right.$ of 2.0$)$. Other authors have shown that $a g g R$ is maximally expressed during the exponential growth phase (Morin et al., 2010) when cells were grown at $37^{\circ} \mathrm{C}$ in DMEM medium plus glucose. Most likely, the nature of these growth media (rich versus minimal) is underlying these differences.

There exist several examples of (p)ppGpp playing a central role in bacterial persistence and virulence (Pizarro-Cerdá and Tedin, 2004; Song et al., 2004; Thompson et al., 2006; Dalebroux et al., 2010; Gaca et al., 2015). We show in this report this is also the case for the expression of the AggR regulator in the EAEC strain 042. A (p)ppGpp ${ }^{0}$ mutant shows reduced aggR transcription and fails to induce it when cells enter the stationary phase, conditions that can be correlated with high (p)ppGpp levels. AggR expression is similar in the 042 WT strain and in its relA derivative. This suggests that spoT (p)ppGpp synthase activity can generate alarmone levels high enough as to maintain high AggR expression. As predicted because of the modulatory role of AggR, lack of (p)ppGpp can also be correlated with reduced expression of AggR modulated genes, such as aff (see Supplementary Figure S4). (p)ppGpp effects can occur by a variety of mechanisms (as reviewed in Magnusson et al., 2005; Hauryliuk et al., 2015; Steinchen and Bange, 2016). A question that remains to be answered is if (p)ppGpp modulates aggR transcription because its direct effect on RNA polymerase, or indirectly, via (p)ppGpp mediated-induction of RpoS (Figure 7).

(p)ppGpp-dependent upregulation of $a g g R$ and its regulatory cascade can be correlated with a virulence phenotype. We could abolish biofilm formation in strain 042 both by (i) generating a (p)ppGpp ${ }^{0}$ mutant, (ii) by knocking out the aggR gene, and (iii) by curing this strain of the pAA2 plasmid. Biofilm formation can be restored both in an $\operatorname{aggR}$ and in a relA spoT mutant by providing in trans the AggR protein, thus supporting the hypothesis that (p)ppGpp-dependent AggR levels are critical for biofilm formation in strain 042. Previous reports have shown that (p)ppGpp influences the phase-variation of fim promoter in $E$. coli, suggesting a dependence upon (p)ppGpp in type 1 fimbriation and consequently in biofilm formation in both $E$. coli K12 and uropathogenic E. coli strains (Aberg et al., 2006). We show here that the relationship between (p)ppGpp and biofilm formation in the EAEC strain 042 occurs, instead of via type 1 fimbriae, via AggR and AAF/II fimbriae.

The fact that our results show a direct correlation between (p)ppGpp, virulence gene expression (e.g., AafA protein) and biofilm formation in strain 042 can be of interest to find out new strategies to combat bacterial infections caused by EAEC. Taking into account that several from these strains can also display a phenotype of multiple antibiotic resistance, combatting infections caused by these strains requires the development of new strategies. Targeting the bacterial stringent response has been studied in the last years as a new approach to combat bacterial infections and biofilm formation (de la Fuente-Núñez et al., 2014; Gaca et al., 2015). A synthetic peptide was recently proposed to 
specifically disrupt biofilms by inhibiting the stringent response via direct interaction with (p)ppGpp (Andresen et al., 2016). The results presented here suggest that these approaches could also be used to combat multiresistant EAEC-mediated infections.

\section{AUTHOR CONTRIBUTIONS}

$\mathrm{MH}$ and $\mathrm{AJ}$ conceived and designed the experiments and wrote the manuscript. $\mathrm{MH}, \mathrm{AP}, \mathrm{JE}$, and $\mathrm{MB}$ performed the experiments.

\section{FUNDING}

This project was funded by grant BIO2016-76412-C2-1-R (AEI/FEDER, UE) from Ministerio de Economía, Industria y Competitividad and CERCA Programme/Generalitat de Catalunya.

\section{ACKNOWLEDGMENTS}

AP was the recipient of a FPU fellowship from the Ministerio de Educación, Cultura y Deporte. $\mathrm{MB}$ was the recipient of a FI Fellowship from the Generalitat de Catalunya.

\section{REFERENCES}

Aberg, A., Fernández-Vázquez, J., Cabrer-Panes, J. D., Sánchez, A., and Balsalobre, C. (2009). Similar and divergent effects of ppGpp and DksA deficiencies on transcription in Escherichia coli. J. Bacteriol. 191, 3226-3236. doi: 10.1128/JB.01410-08

Aberg, A., Shingler, V., and Balsalobre, C. (2006). (p)ppGpp regulates type 1 fimbriation of Escherichia coli by modulating the expression of the site-specific recombinase FimB. Mol. Microbiol. 60, 1520-1533. doi: 10.1111/j.1365-2958. 2006.05191.x

Aberg, A., Shingler, V., and Balsalobre, C. (2008). Regulation of the fimB promoter: a case of differential regulation by ppGpp and DksA in vivo. Mol. Microbiol. 67, 1223-1241. doi: 10.1111/j.1365-2958.2008.06115.x

Andresen, L., Tenson, T., and Hauryliuk, V. (2016). Cationic bactericidal peptide 1018 does not specifically target the stringent response alarmone (p)ppGpp. Sci. Rep. 6:36549. doi: 10.1038/srep36549

Bielaszewska, M., Mellmann, A., Zhang, W., Köck, R., Fruth, A., Bauwens, A., et al. (2011). Characterisation of the Escherichia coli strain associated with an outbreak of haemolytic uraemic syndrome in Germany, 2011: a microbiological study. Lancet Infect. Dis. 11, 671-676. doi: 10.1016/S1473-3099(11)70165-7

Bolivar, F., Rodriguez, R. L., Greene, P. J., Betlach, M. C., Heyneker, H. L., Boyer, H. W., et al. (1977). Construction and characterization of new cloning vehicles. II. A multipurpose cloning system. Gene 2, 95-113. doi: 10.1016/0378-1119(77) 90000-2

Chaudhuri, R. R., Sebaihia, M., Hobman, J. L., Webber, M. A., Leyton, D. L., Goldberg, M. D., et al. (2010). Complete genome sequence and comparative metabolic profiling of the prototypical enteroaggregative Escherichia coli strain 042. PLoS One 5:e8801. doi: 10.1371/journal.pone.0008801

Cherepanov, P. P., and Wackernagel, W. (1995). Gene disruption in Escherichia coli: TcR and KmR cassettes with the option of Flp-catalyzed excision of the antibiotic-resistance determinant. Gene 158, 9-14. doi: 10.1016/0378-1119(95) 00193-A

Czeczulin, J. R., Balepur, S., Hicks, S., Phillips, A., Hall, R., Kothary, M. H., et al. (1997). Aggregative adherence fimbria II, a second fimbrial antigen mediating aggregative adherence in enteroaggregative Escherichia coli. Infect. Immun. 65, 4135-4145.

\section{SUPPLEMENTARY MATERIAL}

The Supplementary Material for this article can be found online at: https://www.frontiersin.org/articles/10.3389/fmicb. 2018.00717/full\#supplementary-material

FIGURE S1 | Full Western blot membrane showing the AggR-Flag and AafA-Flag expression corresponding to Figure 4, lanes 1 and 3 WT, lanes 2 and 4 relA mutant.

FIGURE S2 | Full Western blot membrane showing the AggR-Flag expression corresponding to Figure 4, lane $1 \mathrm{WT}$ and lane 2 relA spoT mutant.

FIGURE S3 | Full Western blot membrane showing the AafA-Flag expression corresponding to Figure 4, lane $1 \mathrm{WT}$ and lane 2 relA spoT mutant.

FIGURE S4 | Complementation of the double relA spoT mutantion by providing SpoT in trans by using the pBR322-SpoT plasmid. (A) M9 minimal medium agar plate showing that the $\mathrm{ppGpp}{ }^{0}$ strain $(\triangle \mathrm{rel} A \Delta s p o T)$ is able to grow in $\mathrm{M} 9$ medium in the presence of pB322-SpoT plasmid. (B) Western blot showing that AafA-Flag protein expression is increased in the $\Delta$ relA $\Delta$ spoT mutant when SpoT is provided in trans. (1) 042 WT (wild-type strain), (2) 042AafAFlag (wild-type strain expressing AafA-Flag), (3) 042AafAFlagrelAspoT [(p)ppGpp ${ }^{0}$ derivative of 042 strain expressing AafA-Flag], (4) 042AafAFlagrelAspoT pBR322

(AafA-Flag ppGpp ${ }^{0}$ strain with control vector), and (5) 042AafAFlagrelAspoT pBR322-SpoT (AafA-Flag ppGpp ${ }^{0}$ strain expressing SpoT in trans).

TABLE S1 | Oligonucleotides used in this work.

Dalebroux, Z. D., Svensson, S. L., Gaynor, E. C., and Swanson, M. S. (2010). ppGpp conjures bacterial virulence. Microbiol. Mol. Biol. Rev. 74, 171-199. doi: 10.1128/MMBR.00046-09

Datsenko, K. A., and Wanner, B. L. (2000). One-step inactivation of chromosomal genes in Escherichia coli K-12 using PCR products. Proc. Natl. Acad. Sci. U.S.A. 97, 6640-6645. doi: 10.1073/pnas.120163297

de la Fuente-Núñez, C., Reffuveille, F., Haney, E. F., Straus, S. K., and Hancock, R. E. W. (2014). Broad-spectrum anti-biofilm peptide that targets a cellular stress response. PLoS Pathog. 10:e1004152. doi: 10.1371/journal.ppat.1004152

Elias, W. P., Czeczulin, J. R., Henderson, I. R., Trabulsi, L. R., and Nataro, J. P. (1999). Organization of biogenesis genes for aggregative adherence fimbria II defines a virulence gene cluster in enteroaggregative Escherichia coli. J. Bacteriol. 181, 1779-1785.

Ellermeier, C. D., Janakiraman, A., and Slauch, J. M. (2002). Construction of targeted single copy lac fusions using lambda Red and FLP-mediated sitespecific recombination in bacteria. Gene 290, 153-161. doi: 10.1016/S03781119(02)00551-6

Frank, C., Werber, D., Cramer, J. P., Askar, M., Faber, M., an der Heiden, M., et al. (2011). Epidemic profile of Shiga-toxin-producing Escherichia coli O104:H4 outbreak in Germany. . N. Engl. J. Med 365, 1771-1780. doi: 10.1056/ NEJMoa1106483

Gaca, A. O., Colomer-Winter, C., and Lemos, J. A. (2015). Many means to a common end: the intricacies of (p)ppGpp metabolism and its control of bacterial homeostasis. J. Bacteriol. 197, 1146-1156. doi: 10.1128/JB. 02577-14

Guzman, L. M., Belin, D., Carson, M. J., and Beckwith, J. (1995). Tight regulation, modulation, and high-level expression by vectors containing the arabinose PBAD promoter. J. Bacteriol. 177, 4121-4130. doi: 10.1128/jb.177.14.41214130.1995

Hauryliuk, V., Atkinson, G. C., Murakami, K. S., Tenson, T., and Gerdes, K. (2015). Recent functional insights into the role of (p)ppGpp in bacterial physiology. Nat. Rev. Microbiol. 13, 298-309. doi: 10.1038/nrmicro3448

Hooper, D. C., Wolfson, J. S., McHugh, G. L., Swartz, M. D., Tung, C., and Swartz, M. N. (1984). Elimination of plasmid pMG110 from Escherichia coli by novobiocin and other inhibitors of DNA gyrase. Antimicrob. Agents Chemother. 25, 586-590. doi: 10.1128/AAC.25.5.586 
Kaper, J. B., Nataro, J. P., and Mobley, H. L. (2004). Pathogenic Escherichia coli. Nat. Rev. Microbiol. 2, 123-140. doi: 10.1038/nrmicro818

Livak, K. J., and Schmittgen, T. D. (2001). Analysis of relative gene expression data using real-time quantitative PCR and the 2(-Delta Delta C(T)) Method. Methods 25, 402-408. doi: 10.1006/meth.2001.1262

Magnusson, L. U., Farewell, A., and Nyström, T. (2005). ppGpp: a global regulator in Escherichia coli. Trends Microbiol. 13, 236-242. doi: 10.1016/j.tim.2005. 03.008

Maisonneuve, E., Castro-Camargo, M., and Gerdes, K. (2013). (p)ppGpp controls bacterial persistence by stochastic induction of toxin-antitoxin activity. Cell 154, 1140-1150. doi: 10.1016/j.cell.2013.07.048

Mayer, C. L., Leibowitz, C. S., Kurosawa, S., and Stearns-Kurosawa, D. J. (2012). Shiga toxins and the pathophysiology of hemolytic uremic syndrome in humans and animals. Toxins 4, 1261-1287. doi: 10.3390/toxins4111261

Miller, J. H. (1992). A Short Course in Bacterial Genetics: A Laboratory Manual and Handbook for Escherichia coli and Related Bacteria. Cold Spring Harbor, NY: Cold Spring Harbor Laboratory Press.

Morin, N., Santiago, A. E., Ernst, R. K., Guillot, S. J., and Nataro, J. P. (2013). Characterization of the AggR regulon in enteroaggregative Escherichia coli. Infect. Immun. 81, 122-132. doi: 10.1128/IAI.00676-12

Morin, N., Tirling, C., Ivison, S. M., Kaur, A. P., Nataro, J. P., and Steiner, T. S. (2010). Autoactivation of the AggR regulator of enteroaggregative Escherichia coli in vitro and in vivo. FEMS Immunol. Med. Microbiol. 58, 344-355. doi: 10.1111/j.1574-695X.2010.00645.x

Nakanishi, N., Abe, H., Ogura, Y., Hayashi, T., Tashiro, K., Kuhara, S., et al. (2006). ppGpp with DksA controls gene expression in the locus of enterocyte effacement (LEE) pathogenicity island of enterohaemorrhagic Escherichia coli through activation of two virulence regulatory genes. Mol. Microbiol. 61, 194-205. doi: 10.1111/j.1365-2958.2006.05217.x

Nataro, J. P., Deng, Y., Cookson, S., Cravioto, A., Savarino, S. J., Guers, L. D., et al. (1995). Heterogeneity of enteroaggregative Escherichia coli virulence demonstrated in volunteers. J. Infect. Dis. 171, 465-468. doi: 10.1093/infdis/171. 2.465

Nataro, J. P., and Kaper, J. B. (1998). Diarrheagenic Escherichia coli. Clin. Microbiol. Rev. 11, 142-201.

Nataro, J. P., Kaper, J. B., Robins-Browne, R., Prado, V., Vial, P., and Levine, M. M. (1987). Patterns of adherence of diarrheagenic Escherichia coli to HEp-2 cells. Pediatr. Infect. Dis. J. 6, 829-831. doi: 10.1097/00006454-198709000-00008

Nataro, J. P., Scaletsky, I. C., Kaper, J. B., Levine, M. M., and Trabulsi, L. R. (1985). Plasmid-mediated factors conferring diffuse and localized adherence of enteropathogenic Escherichia coli. Infect. Immun. 48, 378-383.

Nataro, J. P., Yikang, D., Yingkang, D., and Walker, K. (1994). AggR, a transcriptional activator of aggregative adherence fimbria I expression in enteroaggregative Escherichia coli. J. Bacteriol. 176, 4691-4699. doi: 10.1128/jb. 176.15.4691-4699.1994

Okeke, I. N., Wallace-Gadsden, F., Simons, H. R., Matthews, N., Labar, A. S., Hwang, J., et al. (2010). Multi-locus sequence typing of enteroaggregative
Escherichia coli isolates from Nigerian children uncovers multiple lineages. PLoS One 5:e14093. doi: 10.1371/journal.pone.0014093

Pizarro-Cerdá, J., and Tedin, K. (2004). The bacterial signal molecule, ppGpp, regulates Salmonella virulence gene expression. Mol. Microbiol. 52, 1827-1844. doi: 10.1111/j.1365-2958.2004.04122.x

Sambrook, J. (2001). Molecular Cloning: A Laboratory Manual, 3rd Edn, Vol. 3. Cold Spring Harbor, NY: Cold Spring Harbor Laboratory Press.

Santiago, A. E., Ruiz-Perez, F., Jo, N. Y., Vijayakumar, V., Gong, M. Q., and Nataro, J. P. (2014). A large family of antivirulence regulators modulates the effects of transcriptional activators in Gram-negative pathogenic bacteria. PLoS Pathog. 10:e1004153. doi: 10.1371/journal.ppat.1004153

Sheikh, J., Czeczulin, J. R., Harrington, S., Hicks, S., Henderson, I. R., Le Bouguénec, C., et al. (2002). A novel dispersin protein in enteroaggregative Escherichia coli. J. Clin. Invest. 110, 1329-1337. doi: 10.1172/JCI 16172

Sheikh, J., Hicks, S., Dall'Agnol, M., Phillips, A. D., and Nataro, J. P. (2001). Roles for Fis and YafK in biofilm formation by enteroaggregative Escherichia coli. Mol. Microbiol. 41, 983-997. doi: 10.1046/j.1365-2958.2001.02512.x

Song, M., Kim, H.-J., Kim, E. Y., Shin, M., Lee, H. C., Hong, Y., et al. (2004). ppGpp-dependent stationary phase induction of genes on Salmonella pathogenicity island 1. J. Biol. Chem. 279, 34183-34190. doi: 10.1074/jbc.M3134 91200

Steinchen, W., and Bange, G. (2016). The magic dance of the alarmones (p)ppGpp. Mol. Microbiol. 101, 531-544. doi: 10.1111/mmi.13412

Taylor, R. G., Walker, D. C., and McInnes, R. R. (1993). E. coli host strains significantly affect the quality of small scale plasmid DNA preparations used for sequencing. Nucleic Acids Res. 21, 1677-1678. doi: 10.1093/nar/21.7.1677

Thompson, A., Rolfe, M. D., Lucchini, S., Schwerk, P., Hinton, J. C. D., and Tedin, K. (2006). The bacterial signal molecule, ppGpp, mediates the environmental regulation of both the invasion and intracellular virulence gene programs of Salmonella. J. Biol. Chem. 281, 30112-30121. doi: 10.1074/jbc. M605616200

Uzzau, S., Figueroa-Bossi, N., Rubino, S., and Bossi, L. (2001). Epitope tagging of chromosomal genes in Salmonella. Proc. Natl. Acad. Sci. U.S.A. 98, 15264-15269. doi: 10.1073/pnas.261348198

Conflict of Interest Statement: The authors declare that the research was conducted in the absence of any commercial or financial relationships that could be construed as a potential conflict of interest.

Copyright (c) 2018 Hüttener, Prieto, Espelt, Bernabeu and Juárez. This is an openaccess article distributed under the terms of the Creative Commons Attribution License (CC BY). The use, distribution or reproduction in other forums is permitted, provided the original author(s) and the copyright owner are credited and that the original publication in this journal is cited, in accordance with accepted academic practice. No use, distribution or reproduction is permitted which does not comply with these terms. 